\title{
Redes de Investigación Ecológica y Socio-Ecológica a Largo Plazo (LTER y LTSER) en Iberoamérica: Los casos de México y España
}

\author{
LTER and LTSER in Ibero-America: The cases of Mexico and Spain
}

\author{
MANUEL MAASS ${ }^{1, ~}{ }^{*}$, RICARDO DÍAZ-DELGADO ${ }^{2}$, PATRICIA BALVANERA ${ }^{1}$, ALICIA CASTILLO ${ }^{1}$ \\ \& ANGELINA MARTÍNEZ-YRÍZAR ${ }^{3}$ \\ ${ }^{1}$ Centro de Investigaciones en Ecosistemas (CIEco), Universidad Nacional Autónoma de México (UNAM), Campus \\ Morelia. A.P. 27-3 Sta. Ma. de Guido, Morelia, Michoacán, México 58090 \\ ${ }^{2}$ Estación Biológica de Doñana, Consejo Superior de Investigaciones Científicas, c/ Américo Vespuccio s/n, Isla de la \\ Cartuja, Sevilla, 41092, España \\ ${ }^{3}$ Instituto de Ecología, Unidad Regional de Hermosillo, Universidad Nacional Autónoma de México (UNAM). A. P. 1354, \\ Hermosillo, Sonora, México 83000 \\ *Autor correspondiente: maass@oikos.unam.mx
}

\begin{abstract}
RESUMEN
Las profundas diferencias económicas entre los países, combinadas con igualmente diferentes contextos históricos, sociales y culturales complican enormemente la organización de procesos de investigación científica a escala regional y global. Los diferentes países miembros de la Red Internacional de Investigación Ecológica a Largo Plazo (ILTER) han armado sus propias redes atendiendo a las necesidades, características y restricciones nacionales, pero con miras a participar de manera firme en el proceso internacional. En este artículo se presenta una reseña pormenorizada de las experiencias de dos países iberoamericanos, México y España, en su proceso de conformación de redes LTER nacionales. Los países comparten muchas similitudes culturales, pero con algunas diferencias importantes que han determinado la forma particular en que cada uno de ellos ha diseñado e implementado sus propias redes LTER. Se aborda para ambos casos el proceso de conformación de sus Redes Nacionales en el contexto de su entorno internacional. Asimismo, describe brevemente la manera como ambos grupos están participando en un primer esfuerzo de la red ILTER por detonar un proyecto de colaboración científica a nivel internacional sobre las relaciones existentes entre los motores socioeconómicos y los servicios ambientales de los ecosistemas.
\end{abstract}

Palabras clave: Chamela, Doñana, LTER-España, Mex-LTER, servicios ecosistémicos.

\begin{abstract}
The profound differences between countries, combined with equally diverse historical, social and cultural contexts enormously complicates the organization of scientific research processes at the regional and global scales. The different member countries of the International Long-Term Ecological Research (ILTER) initiative have created their own networks, taking into account their needs, characteristics and national limitations, but with the vision of participating in a strong way in the international processes. In this article, we present a detailed review of the experience of two Ibero-American countries, Mexico and Spain, in their process of forming LTER networks. These two countries share many cultural similarities, but with some important differences that have determined the specific form that each has designed and implemented for their own networks. We address in both cases the process of conforming national networks in the international context, and at the same time we describe briefly the way that each group has participated in the first effort of the ILTER to coordinate a scientific project at the international level regarding the existing relationships between socio-economic drivers and ecosystem services.
\end{abstract}

Key words: Chamela, Doñana, ecosystem services, LTER-España, Mex-LTER.

\section{INTRODUCCIÓN}

Ante la necesidad de lograr un mejor entendimiento de los procesos ecológicos que ocurren a escalas espaciales y temporales grandes, se han creado en todo el mundo redes de investigación ecológica de largo plazo, conocidas como LTER por sus siglas en inglés "Long Term Ecological Research" (http://www.lternet.edu). La idea detrás de 
las redes LTER es sintonizar entre grupos académicos aproximaciones conceptuales, técnicas y metodológicas que permitan comparar resultados de estudios sobre la dinámica funcional de sistemas ecológicos realizados en ambientes naturales altamente contrastantes (Franklin et al. 1990, Gosz 1996).

Como es de esperar, no solo las condiciones ambientales varían enormemente entre países, sino también los recursos materiales y humanos disponibles para poner en operación estas redes LTER. Las profundas diferencias económicas se combinan con igualmente diferentes contextos históricos, sociales y culturales entre países, complicando enormemente la organización de procesos de investigación científica a escala regional y global. El reto de este tipo de iniciativas es cómo lograr avanzar de manera armónica a nivel internacional con tal disparidad de recursos y contrastes socioculturales, por lo que resulta indispensable identificar o crear estrategias para avanzar exitosamente en el desarrollo de la Red Internacional de Investigación Ecológica a Largo Plazo (ILTER por sus siglas en inglés, http://www.ilternet.edu). Solo reconociendo las disparidades en un marco de respeto mutuo a las diferencias culturales será posible ir consolidando una iniciativa mundial de monitoreo y experimentación de sistemas socioecológicos.

Los diferentes países miembros de la ILTER han armado sus propias redes atendiendo a las necesidades, características y restricciones nacionales, pero con miras a participar de manera firme al esfuerzo internacional (ILTER 2000, Parr 2010). En las siguientes líneas se presentan las experiencias de dos países iberoamericanos, México y España, los cuales comparten muchas similitudes culturales, pero con algunas diferencias importantes que han determinado la forma particular en que cada uno de ellos ha diseñado e implementado sus propias redes LTER. Asimismo, y a manera de ejemplo, se reseña la participación de ambos países en el primer esfuerzo de la ILTER por detonar un proyecto de colaboración a nivel internacional sobre servicios ambientales.

\section{EL CASO DE MEXICO}

\section{Iniciativa LTER en México}

La Red Mexicana de Investigación Ecológica a Largo Plazo (Red Mex-LTER; http:// www.mexlter.org.mx) está conformada por 10 grupos académicos que cubren todo el país. Fue creada en octubre de 2004 como una iniciativa académica destinada a fomentar una forma de investigación que permita abordar, de manera interdisciplinaria, el estudio de fenómenos ecológicos a escalas espaciales y temporales amplias (Burgos et al. 2007, Maass et al. 2008). La agenda de investigación de esta Red fue definida con base en los grandes temas de la agenda nacional, y en un análisis de las posibilidades del país para contribuir a la agenda internacional. Las siete Áreas Temáticas identificadas y aplicables al estudio de los ecosistemas naturales terrestres, acuáticos y ecosistemas manejados de México son: i) Patrones y control de la productividad primaria en los ecosistemas; ii) Patrones y control de la dinámica del agua, carbono y nutrientes en los ecosistemas; iii) Papel de la biodiversidad en la estructura y funcionamiento de los ecosistemas; iv) Patrones y frecuencia de las perturbaciones en los ecosistemas; v) Efecto del cambio climático en la estructura y funcionamiento de los ecosistemas; vi) Interacciones al nivel de interfase entre ecosistemas manejados y naturales; y vii) Definición de criterios para el manejo y conservación de los ecosistemas.

También, y siguiendo un mismo criterio de empatar el trabajo de la Red Mex-LTER con la agenda nacional y los intereses de la ILTER, se identificaron siete tópicos de monitoreo que acompañan a las Áreas Temáticas de investigación. Estos incluyen: aspectos de clima; características del suelo; flujos y balances de agua, energía y nutrientes; biodiversidad; abundancia de especies claves; productividad primaria; y cambios en el uso del suelo. Para cada uno de estos tópicos de monitoreo se definieron cuatro niveles de aproximación. Un primer nivel básico que todos los grupos deben cumplir para ser aceptados en la Red; un segundo nivel que debe cubrirse desde el primer año de incorporación a la Red; un tercer nivel que se requiere completar durante los primeros cinco 
años de participación en la Red; y un cuarto nivel definido con base en las condiciones óptimas de monitoreo. Este último nivel no tiene un tiempo perentorio para ser cubierto; sin embargo, se define claramente hacia dónde se quiere dirigir la Red, a fin de que se oriente a aquellos grupos que tienen oportunidad de alcanzar este nivel óptimo de monitoreo. La definición de los niveles 2,3 y 4 obedece a la importancia del parámetro y la dificultad técnica de medirlo. El nivel 2 se refiere a procesos fácilmente medibles y muy importantes para entender la dinámica funcional del ecosistema como, por ejemplo, la lluvia, algún indicador de productividad (e.g., la caída de hojarasca) y los niveles de nutrientes en el suelo. El nivel 3 se refiere a procesos que requieren mayor experiencia técnica, así como la adquisición de equipamiento, por ejemplo, la variación espacial del clima, los flujos de agua en los ríos, niveles de pozos, metales pesados, etc. Por último, el nivel 4 se refiere a variables que requieren equipos más sofisticados y una alta experiencia técnica, como torres climatológicas para medir flujos de carbono, evapotranspiración del dosel y balances de energía.

Dada la participación multiinstitucional de grupos que difieren en tiempos de consolidación académica e infraestructura, es de esperar que a la fecha no todos los grupos cultivan las siete Áreas Temáticas de investigación, ni cubren los siete tópicos de monitoreo. Sin embargo, al ingresar a la Red todos adquieren el compromiso de trabajar para lograrlo. Se requiere cubrir un mínimo de cuatro de las siete Áreas Temáticas y establecer el compromiso de seguir el programa progresivo de monitoreo.

El trabajo dentro de la Red Mex-LTER está orientado a promover la colaboración académica en el marco de proyectos de investigación conjuntos. Sin embargo, la inercia para el arranque de redes ha sido enorme en un país con severas limitaciones económicas, deficientes cuadros académicos (particularmente en materia de investigación ecosistémica) y un sistema científico que durante décadas promovió la competencia y el trabajo individual a costa de los procesos de colaboración académica y el trabajo interdisciplinario. Es por ello que para el armado de proyectos de colaboración se diseñó una estrategia a tres niveles de integración. En un primer nivel, la Red promueve y facilita que sus grupos, de manera individual, continúen haciendo su investigación de largo plazo que los identificó como potenciales miembros de la Red. El segundo nivel de colaboración se detona identificando intereses comunes entre varios de los grupos, ya sea por su proximidad geográfica, por un interés en contrastar condiciones ecológicas o por su afinidad institucional. Sin embargo, el nivel de colaboración que más interesa a la Red es aquel en el que todos los grupos participan en lo que se denominan "Proyectos Estratégicos". Estos proyectos constituyen un verdadero reto académico, dada la complejidad que significa obtener los recursos y sintonizar los intereses, habilidades y metodologías de tantos y tan contrastantes grupos académicos. La clave está en identificar un tema de investigación de interés común y simplificar los requerimientos a un nivel en el que todos puedan participar. Las sinergias que surgen del trabajo colectivo son tan enriquecedoras, que la aparente simplicidad del planteamiento individual (lo que tiene que hacer cada grupo) queda superada por el trabajo de integración que un proyecto de esta naturaleza genera.

Actualmente la Red Mex-LTER trabaja en la conformación de varios proyectos estratégicos, alrededor de las Áreas Temáticas de su agenda de investigación. El proyecto más adelantado se centra en aspectos relacionados con el tema del agua, que por ser fundamental en la dinámica de todo ecosistema, hace factible y viable el planteamiento de un proyecto estratégico en el que todos los grupos de la red puedan participar (Rivera et al. 2008). Una idea central del proyecto es que el manejo apropiado del agua detona importantes procesos de desarrollo económico y social. El proyecto intenta contestar ¿cuál es la capacidad de resiliencia de los principales ecosistemas naturales existentes en México, medida en términos de su funcionamiento hidrológico? Detrás de esta pregunta, que solo puede contestarse con investigación de largo plazo, está el hecho de que el ser humano compite, de manera muy exitosa, con los ecosistemas naturales por los recursos hídricos disponibles. Se trata de identificar qué 
tanto podemos extraer, contaminar o cambiar el régimen hídrico de un ecosistema dado sin que este pierda su integridad funcional a tal grado que genere un cambio de estado el cual no pueda proporcionar los servicios ecosistémicos que requerimos como sociedad. Aunque se trata de un proyecto de largo plazo, se han identificado metas a corto plazo (tres años), entre ellas, la elaboración de un primer análisis integrado sobre los requerimientos hidrológicos de los principales ecosistemas naturales de México. Este primer análisis dista de ser completo, pero será una muy importante contribución al programa nacional de pago por servicios ambientales el cual, está basado en información científica fragmentada, que ponen en duda el cumplimiento de los supuestos de los modelos empleados.

Es importante enfatizar que el proceso de construcción de la Red Mex-LTER no ha sido fácil. Pasaron varios años hasta poner de acuerdo a un grupo de 10 investigadores que concibieron y estructuraron la propuesta para definir los objetivos, metas y reglas de operación de la Red. Una vez creada oficialmente, han sido necesarios varios años para sintonizar el trabajo colectivo e implementar la estrategia planteada. Aspectos geográficos, históricos y culturales han contribuido en mayor o menor grado en el proceso de arranque, del cual abordaremos los aspectos más importantes.

No obstante el carácter de corto plazo que domina en la mayoría de los estudios que se realizan en las estaciones de campo, con el tiempo el conocimiento se fue acumulando y concentrando en las zonas donde se ubican estas estaciones de investigación, convirtiéndolas en candidatas idóneas para armar las redes LTER. En México, sin embargo, más que armar una red de estaciones de campo, el interés fue armar una red de grupos de investigación. Esto es, grupos académicos trabajando de manera colectiva hacia el entendimiento de procesos ecológicos complejos, solo abordables con esfuerzos conjuntos, integrados y con una fuerte capacidad de trabajo en equipo. Naturalmente, algunas de las estaciones de campo fueron materia prima para la conformación de grupos.

La Red Mex-LTER no debe verse como una red de monitoreo ambiental, sino como una red de grupos académicos que utilizan el seguimiento de largo plazo de variables ecológicas claves como una herramienta de trabajo y no como un fin. La idea ha sido identificar o crear grupos mexicanos capaces no solo de recopilar datos, sino de plantearse preguntas de investigación, diseñar los protocolos de muestreo, analizar la información generada y publicar sus resultados. Con esta estrategia se pretende fortalecer a los grupos orientados en la misma dirección, más que convertirse en meros generadores de datos bajo la dirección de grupos extranjeros, situación muy frecuente en países con poco desarrollo científico local. De lo que se trata es que al reconocer que se tienen carencias académicas, se busquen los mecanismos que permitan resolverlas mediante un ambicioso programa de formación de recursos humanos.

Este planteamiento de construir grupos académicos soportados esencialmente por investigadores nacionales, ha hecho el proceso de creación de la Red Mex-LTER más lento, pero sobre terreno más firme. Se aprecia la colaboración extranjera y el proceso de conformación de grupos se apoya fuertemente en ella, pero se asegura un liderazgo, crecimiento y coordinación en manos de los investigadores locales.

Hay que reconocer que, no obstante en México existen investigadores de alto nivel académico, no hay una tradición de trabajo colectivo. El armado de redes de investigación no solo requiere resolver esta problemática y buscar metas comunes, sino también resolver aspectos económicos y técnicos. A este respecto, el Consejo Nacional de Ciencia y Tecnología (CONACYT) ha sido un gran aliado. Desde su formación en el 2004, la Red ha recibido apoyo económico por parte del Consejo para operar una oficina y lograr una adecuada movilidad académica. Asimismo, y a través de su Programa de Apoyo a la Investigación Básica, obtuvo financiamiento para arrancar el proyecto estratégico sobre ecohidrología antes mencionado. Más aún, la actual administración del CONACYT ha dado un paso sin precedentes en lo que se refiere a estimular la investigación colectiva en México al convocar este año, a la conformación de 14 redes temáticas de investigación a nivel nacional. La Red Mex-LTER no solo contribuyó 
a la detonación de este proceso, sino que además su experiencia ha sido muy útil en la creación de algunas de estas redes, particularmente la de Medio Ambiente y Sustentabilidad de la que forma parte. Otros aliados fundamentales en el armado de la Red Mex-LTER han sido entidades gubernamentales y académicas, en particular el Instituto Nacional de Ecología (INE), la Comisión Nacional para el Uso de la Biodiversidad (CONABIO), el Centro de Investigaciones en Ecosistemas de la UNAM y el Instituto de Ecología, A.C. Su respaldo moral, sustento económico y apoyo en especie (e.g., instalaciones y salarios) han sido indispensables para el despegue y operación de la Red Mex-LTER.

Por último, nos referiremos al trabajo interdisciplinario que realiza la Red MexLTER. De igual forma que la gran biodiversidad ha definido rutas particulares del desarrollo de las ciencias biológicas en México, esta diversidad biológica y de paisajes también congrega una gran diversidad de etnias y grupos culturales en el país, lo que ha contribuido al importante desarrollo que han tenido las ciencias sociales. Desafortunadamente, estas y las ciencias ambientales tienen poca interacción e integración institucional, de tal forma que los académicos de ambas disciplinas poco colaboran entre sí. No obstante que la Red Mex-LTER se conformó como una red de corte ecológico, desde su inicio ha existido un interés por incorporar aspectos sociales, esto es, de transitar de una LTER hacia una LTSER (Long-Term Socio-Ecological Research, Haberl et al. 2006, Jardel et al. 2008). En un esfuerzo por impulsar la colaboración entre ambos campos del conocimiento, la Red Mex-LTER ha identificado áreas temáticas de investigación de corte socioambiental, bajo las siguientes preguntas: ¿cuáles son los contornos de vulnerabilidad en la transformación de los Socio-Eco-Sistemas?; ¿cómo es que cambios demográficos afectan las decisiones y prácticas de manejo de los Socio-Eco-Sistemas local y regional?; ¿cómo son las relaciones/valoraciones económicas en cuanto a bienes y servicios brindados por los Socio-Eco-Sistemas?; ¿cómo están estructuradas y cómo funcionan las instituciones sociales (gobernanza) locales para el manejo de los Socio-Eco-Sistemas?; ¿qué papel desempeña la información y la educación (formal, no formal e informal) en las decisiones sobre manejo de Socio-EcoSistemas?; y ¿cuáles es la cultura ambiental (percepciones y expectativas) de los pobladores con respecto a la transformación de sus Socio-Eco-Sistemas?

El proceso ha comenzado pero tenemos un arduo camino por delante. No solo son contados los académicos del campo de las ciencias sociales interesados en incorporase de lleno a la Red, sino también algunos miembros de la Red no están convencidos por convertirse en una LTSER. Sin embargo, el cuerpo directivo de la Red tiene una firme convicción de la necesidad de dar dicho paso y muchas de las actividades grupales están encaminadas a lograrlo.

\section{EL CASO DE ESPAÑA}

\section{La Iniciativa LTSER en Europa}

La red LTER-Europa (http://www.1tereurope.net) fue constituida en 2007, uniendo las dos redes regionales de ILTER de Europa Occidental y Oriental. En la actualidad está conformada por 18 redes nacionales. Esta red emerge como propuesta para abordar las conclusiones de la Evaluación de los Ecosistemas del Milenio (MEA 2005), el Informe Global de Medio Ambiente (UNEP 2007) y varios informes de la Agencia Europea de Medio Ambiente (EEA 2005, 2007), que plantean la necesidad de integrar naturaleza y sociedad para afrontar los retos de gestión de la naturaleza. Nace como una red de "última generación" compuesta por sitios tradicionales LTER (escala local) y plataformas LTSER. Estas plataformas han sido propuestas en regiones propicias para la investigación socioecológica y su implementación busca una eficiente gestión y conservación de la naturaleza (Mirtl \& Krauze 2007). La constitución de LTER-Europa en 2007 conllevó la propuesta de 16 plataformas LTSER piloto según los criterios propuestos por Ohl et al. (2007). Durante 2008, se han propuesto cuatro nuevas plataformas LTSER en Europa, poniendo de manifiesto el éxito de la iniciativa con un total de 20 regiones LTSER. Todas ellas 
se han establecido en sitios LTER tradicionales. Un reciente estudio realizado en el marco de LTER-Europa ha puesto de manifiesto los vacíos existentes en la cobertura de las unidades socioecológicas de Europa por las regiones LTSER establecidas (Fig. 1). La estratificación en unidades socioecológicas se obtuvo a partir del cruce de las unidades ambientales establecidas por Metzger et al. (2005) y la información socioeconómica asociada a la base de datos de la Agencia Europea de Medio Ambiente empleando los datos del Producto Interior Bruto (PIB), densidad poblacional y el nivel de accesibilidad (densidad de infraestructuras de comunicación). Los resultados muestran las áreas biogeográficas y los contextos socioeconómicos sobrerrepresentados por los sitios LTER y las regiones LTSER establecidas y aquellas con baja o nula representación. Sorprendentemente, para las regiones LTSER existe una buena cobertura, si bien la región Mediterránea se encuentra aún poco representada.

\section{LTER-ESPAÑA}

Los orígenes de la red española de investigaciones ecológicas de largo plazo (LTER-España) se remontan a 2003 cuando un grupo de trabajo de los científicos españoles participantes en el IGBP (Internacional Geosphere and Biosphere Programme), funda REDOTE (Red Española de Observaciones Temporales de Ecosistemas). REDOTE fue una iniciativa cuyo objetivo principal era identificar, recopilar y organizar en una base

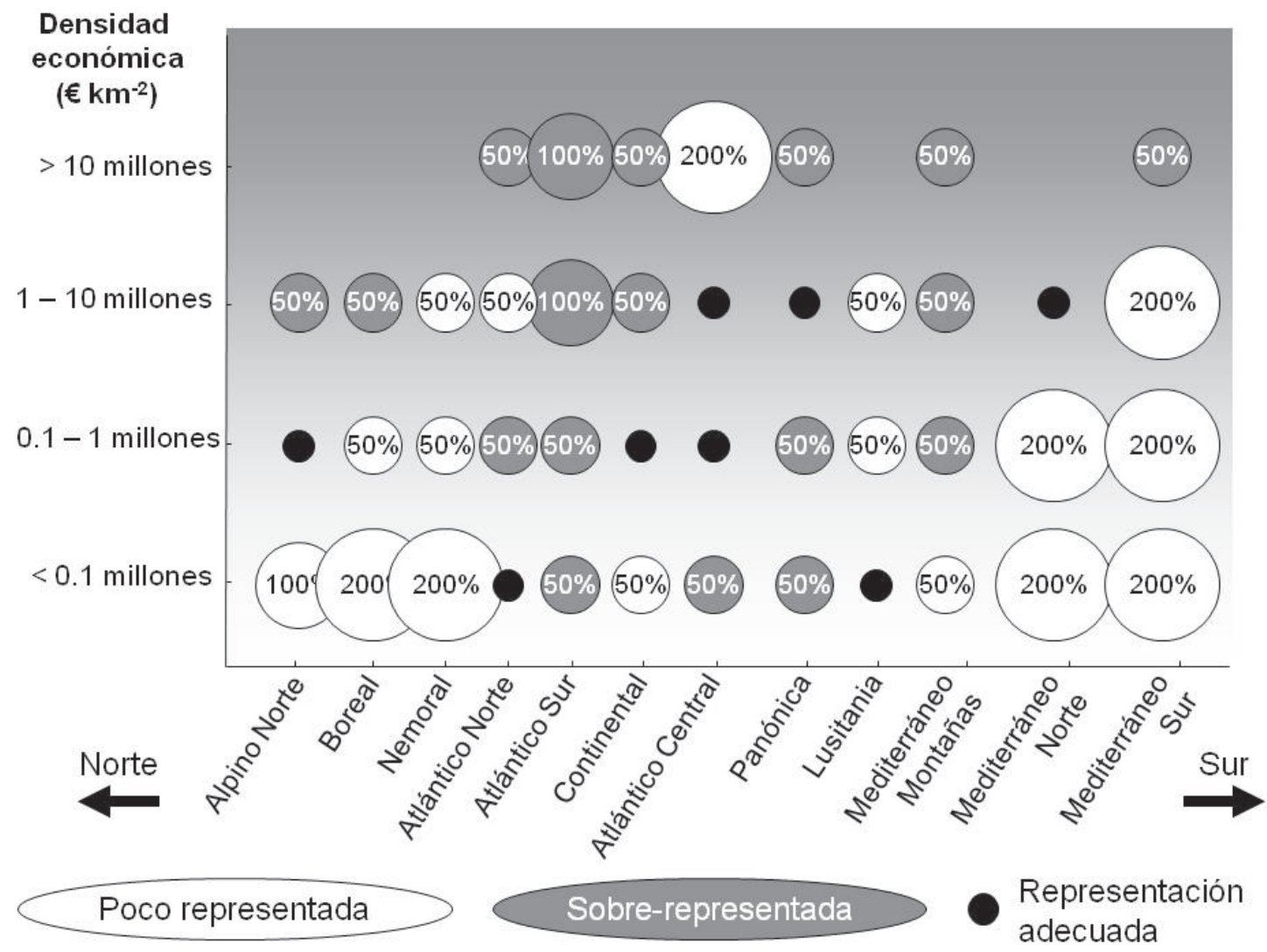

Fig. 1: Nivel de representatividad de los sitios LTER y regiones LTSER en la red LTER-Europa con respecto a las condiciones socioeconómicas (ingresos en euros por $\mathrm{km}^{2}$ ) frente al rango de regiones biogeográficas (modificado a partir de Metzger et al. 2005).

Level of representation of LTER sites and LTSER regions in the Europe LTER Network with respect to the socioeconomic conditions (income in Euros per $\mathrm{km}^{2}$ ) across the range of biogeographic regions (modified from Metzger et al. 2005). 
de datos todas las actividades de investigación ecológica a largo plazo y los programas asociados relevantes para el seguimiento de procesos naturales y la comprensión del Cambio Global y su impacto en los ecosistemas españoles.

Después de varios años de reuniones, talleres e intenso trabajo, REDOTE compiló un documento sobre esta iniciativa pionera acerca de sus miembros y la información a largo plazo disponible, accesible a través de su página web (http://www.redote.org). Desde entonces, REDOTE ha sido elemental para la selección de los sitios candidatos para conformar LTEREspaña y en la identificación de los grupos científicos destinados a coordinar las actividades relacionadas con LTER tanto dentro de España como integrados en las iniciativas europea y global. Desde aquel momento, esta protorred española puso de manifiesto la firme intención de convertirse en miembro reconocido de ILTER.

Al mismo tiempo, el Consejo Superior de Investigaciones Científicas (CSIC) comenzó a apoyar la iniciativa involucrándose en la Red Europea de Excelencia ALTER-Net. El CSIC, con 28 centros de investigación en el área de Recursos Naturales y más de 300 científicos en plantilla ha conducido el proceso LTER, impulsado principalmente por la Estación Biológica de Doñana (http:// www.ebd.csic.es), el Instituto de Recursos Naturales (http://www.ccma.csic.es), el Instituto de Recursos Naturales y Agrobiología de Sevilla (http://www.irnase.csic.es) y el Centro de Estudios Avanzados de Blanes (http://www.ceab.csic.es). Además de la participación del CSIC en ALTER-Net, la reciente creación de LTER-Europa ha fomentado intensamente la participación de los países europeos en la creación de redes LTER/LTSER más eficaces y ha promovido definitivamente el proceso LTER español.

El plan estratégico de LTER-España ha tenido que hacer frente a la peculiar estructura de gobierno del estado autonómico español dada la descentralización existente en las políticas relativas a investigación $\mathrm{y}$ conservación del medio ambiente. El primer Comité Ejecutivo provisional de LTER-España decidió adoptar una estrategia ascendente ("bottom-up"), priorizando las áreas protegidas como candidatos potenciales, especialmente los Parques Nacionales (PPNN). Este criterio se justifica desde diferentes perspectivas. Por un lado, desde el año 2000 el Ministerio de Medio Ambiente financia un programa competitivo anual de investigación en PPNN. Por otro lado, el Organismo Autónomo de PPNN (OAPN, dependiente de ese ministerio) se encuentra en pleno proceso de desarrollo e implementación de un programa de seguimiento ecológico a largo plazo en estas zonas protegidas que coincide plenamente con los objetivos de LTER-España. Por último, y no menos importante, los PPNN requieren y demandan para su gestión programas científicos asociados de seguimiento ecológico a largo plazo que permitan ofrecer herramientas de manejo ante los procesos de cambio global y la problemática específica de cada área protegida.

Es precisamente durante el año 2007 cuando comienza la transferencia de la gestión de los PPNN desde el gobierno central a los gobiernos autonómicos. Esta coyuntura política contribuye a solicitar por parte de LTER-España a los sitios candidatos el respaldo y apoyo institucional de los gobiernos autonómicos correspondientes mediante memorandos de entendimiento de acuerdo con las recomendaciones de LTER-Europa. Estos documentos recogen el compromiso explícito en financiación, personal, infraestructura, intercambio de datos, disponibilidad de información en inglés entre otros. Este apoyo se revela como esencial para garantizar la implicación de los gobiernos regionales en la financiación y soporte estructural de la investigación y el seguimiento ecológico a largo plazo en los sitios propuestos bajo su administración y gestión.

Los grupos de investigación asociados a los sitios candidatos han manifestado desde el principio su voluntad y firme intención de participar en el proceso de implementación de la red española y se han comprometido en su sostenimiento. Diferentes instituciones de investigación, incluyendo varios centros del CSIC, universidades y centros adscritos, así como diferentes redes de investigación y asociaciones como la Asociación Española de Ecología Terrestre (AEET) y la Red Nacional de la Ciencia del Cambio Global en la Biodiversidad y Ecosistemas Mediterráneos (GLOBIMED), han apoyado desde el 
comienzo el proceso LTER en España, designando para cada sitio candidato un responsable científico. De nuevo, siguiendo las recomendaciones de LTER-Europa, se han designado también responsables de gestión para cada uno de los sitios candidatos, que en la mayoría de los casos coinciden con los directores conservadores de los espacios protegidos.

Así en 2007 se propusieron 10 sitios candidatos para conformar LTER-España, 9 sitios LTER tradicionales y 1 plataforma LTSER. En virtud de su grado de cumplimiento de los criterios establecidos la propuesta fue aprobada mediante un amplio consenso por parte del Comité Ejecutivo provisional. La red de sitios se extiende por las regiones biogeográficas de la Península Ibérica, desde el bioma Eurosiberiano al Mediterráneo árido, desde los ecosistemas marinos a los alpinos, con espacios naturales integrados en matriz urbana hasta las áreas naturales más emblemáticas y menos humanizadas. La inclusión de cuatro Parques Nacionales (Doñana, Sierra Nevada, Aigüestortes e Illas Atlánticas) se basa en el sólido apoyo del OAPN y amplía los beneficios de la red en el contexto de la gestión para mejorar el conocimiento de los procesos ecológicos a largo plazo y la colaboración entre gestores y científicos. La estructura ejecutiva de LTER-España refleja así la peculiaridad del proceso de implementación LTER en España. LTER-España ofrece varias categorías de miembros con objeto de facilitar la integración de la amplia y diversa comunidad de científicos y gestores participando con diferentes niveles de compromiso (comité ejecutivo, comité científico, responsables gestores de sitio, responsables científicos de sitio y miembros interesados).

A lo largo de 2007, las actividades de LTEREspaña de acuerdo con su hoja de ruta consistieron en la formalización del Comité Ejecutivo de la red, la estructura de organización, la creación de redes funcionales entorno a diferentes temáticas imprescindibles, tales como: desarrollo de una base de datos y metadatos común y acceso público a la información, diseño y actualización de la web (http://www.lter-spain.net), tareas de secretariado, definición de las áreas centrales de investigación ecológica a largo plazo y del plan estratégico compartido con la red europea e internacional. Como horizonte se estableció la candidatura de LTER-España para ser aceptado como miembro de ILTER en la reunión anual de 2008 .

Las áreas principales de investigación de LTER-España comúnmente establecidas, de acuerdo con las prioridades de investigación científica por parte de los miembros de la red y con respecto a la problemática de gestión asociada a cada uno de los sitios, son: Función de la diversidad biológica en el funcionamiento de los ecosistemas, en la resiliencia y en su estructura; Patrones y frecuencias de las perturbaciones y su papel en la dinámica de los ecosistemas; Efectos del Cambio Global en el funcionamiento de los ecosistemas y su estructura (identificación de umbrales de cambios irreversibles); Definición de criterios de gestión adaptativa y en la toma de decisiones para la conservación; Investigación socioecológica a largo plazo.

El plan estratégico de LTER-España se concentra en fomentar entre los científicos españoles y los gestores de espacios protegidos la investigación y seguimiento ecológico a largo plazo mediante el empleo de protocolos metodológicos comunes actualizados y consensuados. De este modo, LTER-España pretende promover las actividades de investigación ecológica a largo plazo de forma integrada a través de las áreas principales de investigación de LTER-España y las agendas científicas de LTER-Europa e ILTER, garantizando e impulsando la difusión y la divulgación de la implementación de las actividades de la investigación ecológica a largo plazo.

Otro de los aspectos fundamentales desarrollados por LTER-España ha sido la propuesta de unos parámetros ecológicos mínimos para ser sistemáticamente medidos y cuantificados en cada sitio de la red LTEREspaña con objeto de hacerlos comparables. Estos parámetros se agrupan en cuatro compartimentos: el medio abiótico, los productores primarios, los consumidores y parámetros relacionados con la influencia humana. En la actualidad la red comparte mayoritariamente la adquisición sistemática de los parámetros meteorológicos, de estructura forestal, de estado de las poblaciones de aves e 
invertebrados, de características edáficas, de deposición atmosférica, de fenología y parámetros limnológicos de las masas de agua. Finalmente, en agosto de 2008, LTER-España fue exitosa y formalmente aceptada como miembro de ILTER y por ende de LTEREuropa.

\section{ESTUDIO COMPARADO DE LOS SERVICIOS AMBIENTALES}

El concepto de servicios ecosistémicos ha sido el marco de trabajo que está utilizando el Comité Científico de la Red ILTER en su primer esfuerzo por detonar un proyecto de colaboración a nivel internacional entre sus redes nacionales. El principal objetivo del proyecto es identificar qué tanto las diferencias culturales en la percepción y uso de los servicios ecosistémicos limitan la resiliencia de los socio-eco-sistemas (SES). Esto se pretende lograr comparando el uso de servicios ecosistémicos como función de la percepción de los cambios en el suministro de estos servicios, bajo un mismo tipo de bioma, pero bajo diferentes sistemas culturales. El modelo de trabajo fue desarrollado en 2007 por la Red Americana LTER durante el diseño de su iniciativa estratégica de investigación conocida como ISSE, por sus siglas en inglés "Integrative Science for Society and the Environment" (Collins et al. 2007, Bourgeron 2008)

Tanto México (el sitio Chamela Mex-LTER) como España (la plataforma LTSER-Doñana) colaboran en este proyecto piloto de la ILTER. Como parte del presente análisis en el que se contrastan la forma que México y España han ido implementado su programa LTER, se ha decidido incluir una breve reseña de cómo ambos países están abordando su participación en la iniciativa ISSE.

El ejercicio comienza por la descripción del socioecosistema siguiendo el marco conceptual del ISSE (Fig. 2), incluyendo la identificación de los servicios ecosistémicos más críticos, su dirección de cambio, la percepción que tiene la población de dichos servicios así como los principales factores que inducen estos cambios en la provisión del servicio y las instituciones sociales que permiten su manejo.
La aplicación de la aproximación ISSE en el sitio Chamela Mex-LTER

En el caso particular de Chamela, el socioecosistema bajo análisis lo constituye la cuenca del río Cuiztmala con una superficie un poco mayor a los $1,000 \mathrm{~km}^{2}$ y en el que predominan las selvas bajas caducifolias con alta diversidad biológica, en lomeríos suaves con suelos someros y arenosos (Maass et al. 2005). En la parte alta de la cuenca se desarrollan bosques de pino y de encino, mientras que a lo largo de los cauces de los ríos y las zonas aluviales de la parte media y baja de la cuenca, se desarrolla una selva mediana subcaducifolia sobre suelos planos, profundos y ricos en nutrientes y materia orgánica. El funcionamiento del ecosistema responde en buena medida a los efectos de pulsos de agua producto de la actividad ciclónica y eventos El Niño. Ante la marcada estacionalidad climática, con el $80 \%$ de las lluvias concentradas en tan solo 4-5 meses del año, las plantas responden tirando sus hojas durante la época de sequía, dándole ese carácter deciduo tan conspicuo al bosque tropical seco. La zona ha estado por varias décadas bajo una constante presión de transformación y cambio de uso del suelo, convirtiendo a las selvas bajas en praderas ganaderas y las selvas medianas en tierras de cultivo. Una buena parte de la población se encuentra dispersa en pequeño poblados dentro de la cuenca, pero existe una importante concentración en la parte alta $\mathrm{y}$ baja de la cuenca. Arriba, en los márgenes de la cuenca, se encuentra Villa Purificación, una pequeña ciudad con más de 400 años de historia y una población menor de 5,000 habitantes, y en la parte baja, se han desarrollado pueblos y asentamientos hoteleros con una importante actividad turística a lo largo de la zona costera.

Se han identificado más de 10 grandes tipos de servicios ecosistémicos en esta zona (Maass et al. 2005). La provisión de productos forestales y de un sustrato para el desarrollo de la agricultura y ganadería son los servicios más apreciados por la población local. Asimismo, la belleza escénica es apreciada por locales y visitantes. Sin embargo, las limitaciones hídricas de la zona, el suministro de agua limpia en pozos y ríos está menos 
identificado como servicio del ecosistema, y menos aún el servicio de control de inundaciones.

En el esquema de trabajo del ejercicio ISSE las seis preguntas que determinan el grado de interacción entre los diferentes subsistemas del socioecosistema y sus efectos sobre escenarios futuros (Fig. 2) son: ¿cómo se adapta el ecosistema de bosque tropical seco (para simplificar "bosque") a las presiones de conversión a praderas ganaderas y a los pulsos de lluvia y sequía de corto plazo mediante sus atributos estructurales y funcionales?; ¿cómo la alta diversidad del bosque ayuda o limita la dinámica hidrológica, biogeoquímica y productiva del ecosistema?; ¿qué procesos ecosistémicos (o atributos estructurales) del bosque proveen los diferentes servicios ecosistémicos y a qué escala operan?; ¿qué tanta resiliencia tiene el bosque (i.e., qué tanta perturbación resiste el ecosistema sin cambiar el estado en el que provee los servicios ecosistémicos)?; ¿qué pierde o gana ("tradeoff") la población cuando el bosque es trasformado en praderas ganaderas?; ¿cuál es la percepción que tienen los diferentes usuarios ("stakeholders") de la cuenca de los diferentes servicios ecosistémicos?; ¿cómo responden los usuarios de la cuenca a este conocimiento, o falta de él, en relación a los servicios ecosistémicos?; ¿qué acciones humanas tienen influencia en la frecuencia, magnitud o forma en la que ocurren estos pulsos de agua y presiones de transformación del bosque, y qué factores directos o indirectos determinan estas acciones humanas?; ¿qué intervenciones técnicas, comunicativas o instituciones pueden ser implementadas para mejorar un manejo sustentable del bosque en la cuenca del río Cuitzmala?

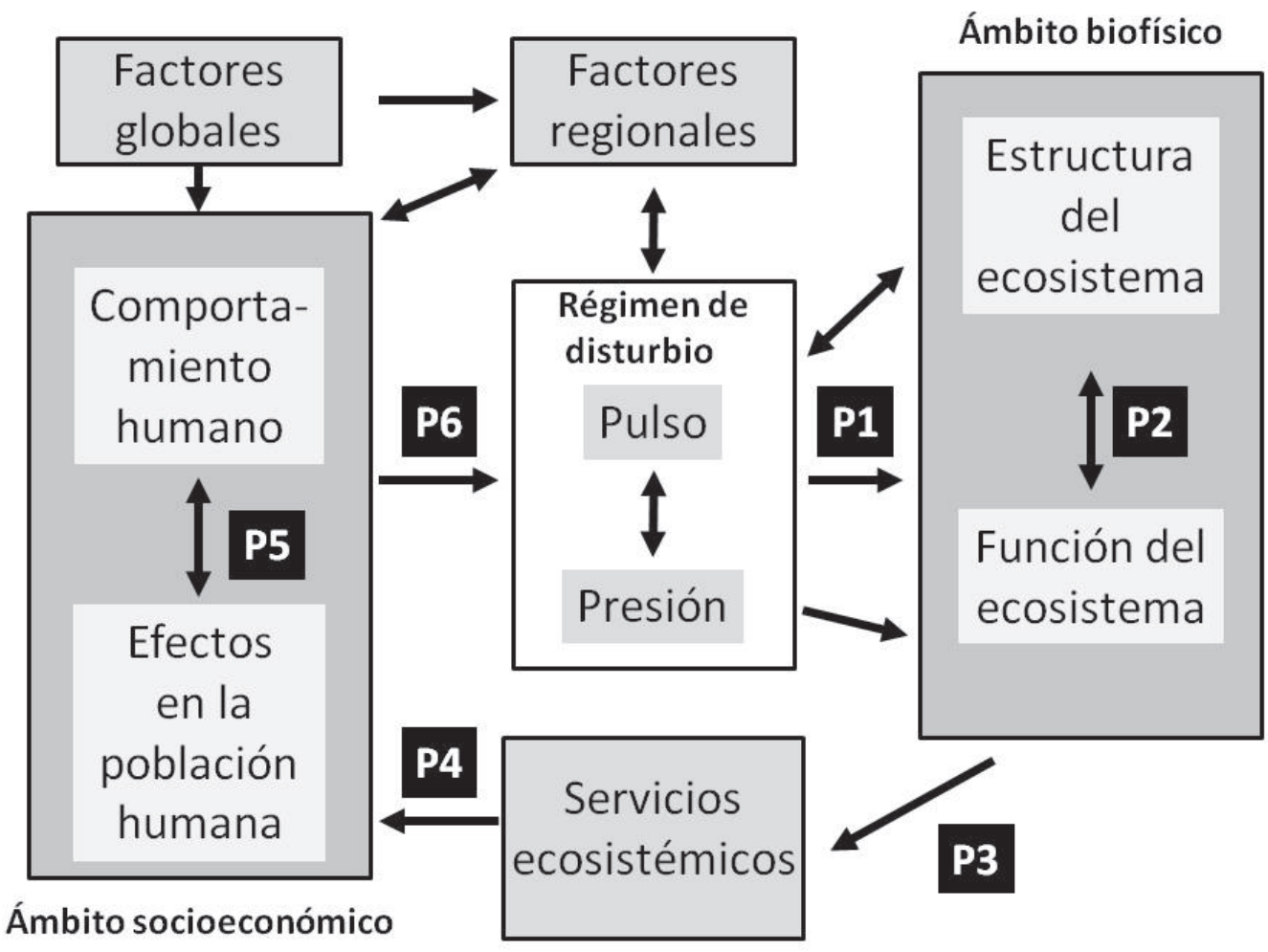

Fig. 2: Modelo de trabajo desarrollado por la Red Americana LTER durante el diseño de su iniciativa estratégica de investigación conocida como ISSE (por sus siglas en inglés "Integrative Science for Society and the Environment”, modificado de Collins et al. 2007). Los cuadros P1 a P6 corresponden a las preguntas que determinan el grado de interacción entre los diferentes subsistemas del socioecosistema.

Working model developed by the US LTER Network during its strategic plan design, known as ISSE (Integrative Science for Society and the Environment, modified from Collins et al. 2007). The boxes P1 to P6 correspond to questions that determine the degree of interaction between the different subsystems of the socio-ecoystem. 
Actualmente la cuenca se encuentra en un estado de degradación caracterizado por un régimen de conversión de bosque a pradera, un proceso de fragmentación del terreno y pérdida de la conectividad hidrológica, una atomización de la tenencia de la tierra, una pérdida de identidad del campesino y un proceso de emigración. A pesar de ello, la cuenca mantiene una razonable provisión de servicios ecosistémicos y baja actividad turística. Los tres posibles escenarios futuros identificados por el Grupo son los siguientes:

(1) Un incremento en las actividades agropastoriles, caracterizado por un aumento en la transformación y fragmentación de terreno, una mayor demanda de servicios ecosistémicos por parte de la población, una menor provisión de estos servicios (excepto la provisión de productos agropecuarios), una pérdida de la conectividad hidrológica, así como una pérdida del potencial turístico.

(2) Un aumento masivo en las actividades turísticas, caracterizado por una disminución en las tasas de conversión del suelo, un incremento en las demanda de servicios ecosistémicos, un incremento en la privatización del terreno, un aumento en la pérdida de identidad campesina y la detonación de un proceso de inmigración en la región.

(3) Un manejo sustentable de la cuenca, caracterizado por una conservación y restauración del ecosistema original, un aumento en la provisión de servicios ecosistémicos, un mantenimiento de la identidad campesina, la estabilización de la población y el mantenimiento de un alto potencial turístico.

\section{La aplicación de la aproximación ISSE en la plataforma LTSER Doñana}

Las marismas de Doñana han sufrido dramáticas transformaciones a lo largo del último siglo, a consecuencia de la desecación de las zonas inundables para cultivos, la canalización de los brazos del río Guadalquivir, las medidas de conservación de la biodiversidad y protección frente al vertido tóxico de la mina de Aznalcóllar en el año 1998 (Pain et al. 1998). La superficie ocupada a principios del siglo XX por las marismas de Doñana se estima en $1,500 \mathrm{~km}^{2}$ (Casado \& Montes 1991). En la actualidad se conservan $270 \mathrm{~km}^{2}$ lo que supone una pérdida del $82 \%$ de la superficie original. Además de la pérdida de humedales, la influencia mareal, uno de los principales factores de inundación previo a las transformaciones humanas, fue limitada a partir de 1984 mediante la construcción de un dique a lo largo de la margen derecha del río Guadalquivir denominado "montaña del río". Entre cambios tan dramáticos, el vertido tóxico procedente de la balsa de lodos de la mina de pirita de Aznalcóllar, situada en las proximidades del río Guadiamar tributario de la marisma, ocurrido en abril de 1998, conllevó la puesta en marcha de un ambicioso proyecto de restauración hidrológica de las marismas de Doñana. El proyecto, denominado "Doñana 2005” financió diferentes actuaciones valoradas en 140 millones de euros. Los objetivos principales son la recuperación de la cantidad y calidad de las aguas entrantes a Doñana de acuerdo con una estrategia de restauración adaptativa (Lee 1993, Holling 2002). Este proyecto, que aúna los esfuerzos de diferentes administraciones, pretende recuperar los servicios ambientales o ecosistémicos proporcionados por la marisma como respuesta a los principales motores de cambio y las presiones sobre la biodiversidad en las últimas décadas (Chans \& Díaz-Delgado 2005). Una de las actuaciones más significativas busca recuperar la secular influencia mareal por medio de la eliminación de la "montaña del río". Esta decisión ha sido tomada por el comité científico del proyecto asumiendo los riesgos potenciales de nuevos episodios de contaminación o de expansión de especies invasoras (e.g., la Spartina densiflora Brong, un helófito invasor de carácter mareal que se encuentra confinado en el margen del río Guadalquivir y en otras marismas mareales desplaza eficazmente a la flora nativa, cf. Nieva et al. 2003, Castillo et al. 2000). Esta situación se convierte en un verosímil estado futuro del sistema que puede evaluarse a través de la aproximación metodológica ISSE.

El ejercicio común planteado en el marco de ILTER que emplea la aproximación ISSE pretende determinar a diferentes escalas las relaciones existentes entre los motores socioeconómicos y los servicios ambientales de los ecosistemas, permitiendo evaluar la magnitud y dirección de las presiones sobre los ecosistemas y su biodiversidad, así como la reversibilidad de los procesos. 
En Doñana se han identificado seis servicios ecosistémicos como los más críticos que interaccionan entre sí de la siguiente forma:

Uso para consumo humano de agua subterránea vs. producción alimentos (agricultura intensiva y bajo plástico) vs. turismo masivo de temporada.

Capacidad de regulación de la inundación por parte de la superficie marismeña vs. producción alimentos (arrozal y piscicultura) vs. función de retención de nutrientes y sedimentos.

Regulación de riesgos naturales (meteotsunamis) vs. regulación de inundación por parte de la superficie marismeña vs. turismo masivo de temporada

De todos ellos se seleccionó como el más crítico para el sistema la regulación de inundación y el funcionamiento estuarino. El esquema de trabajo plantea seis cuestiones que determinan el grado de interacción entre los diferentes subsistemas (socialeconómicoecológico) y sus efectos sobre escenarios futuros (Fig. 2). En el caso de la plataforma Doñana-LTSER son: ¿cómo el incremento en la demanda de agua y el tráfico ribereño alterarán la estructura y funcionamiento del ecosistema estuarino?; ¿cómo las comunidades bióticas se ven afectadas por los cambios en el hidroperiodo de inundación, la influencia mareal y las elevadas cargas de sedimentos?; ¿cómo los cambios en el funcionamiento de la marisma afectarán la regulación de inundación, el control estuarino y a la demanda hídrica para los arrozales y la piscicultura?; ¿cómo los cambios en la disponibilidad de agua dulce y en el servicio de regulación de inundación modificarán las políticas de conservación y afectarán a las principales actividades económicas (turismo y agricultura)?; ¿cómo convergirán las políticas de conservación y de desarrollo económico y cómo se regulará la tasa de inmigración de acuerdo con los cambios en el mercado económico?; y ¿cómo la combinación del comercio, la conservación y las políticas de desarrollo turístico influirán en el régimen de inundación de las marismas de Doñana y cuáles serán los principales puntos en común de dichas políticas para un desarrollo sostenible?

En el estado actual, el principal ecosistema de Doñana es una marisma de carácter fluvial, sin influencia mareal ni control estuarino en la desembocadura del Guadalquivir, lo que provoca frecuentemente episodios de extrema turbidez que afectan a las actividades que requieren agua dulce tales como el arrozal o la piscicultura (Bustamante et al. 2009). Debido a la construcción y prolongación de la montaña del río se produce sobreinundación con frecuencia en la marisma de Doñana. Esta configuración reduce el riesgo de exposición a eventos externos pero otorga un grado de aislamiento a la marisma manteniéndola hidrológicamente desconectada. Los posibles escenarios de futuro son:

"Todo sigue igual": Las aguas del río Guadalquivir mantendrán su baja calidad, se evitarán eventos de entrada de contaminantes en la marisma, se limitará la invasión de especies invasoras y se mantendrán hábitats y especies características de humedales dulceacuícolas.

"Restauración de la permeabilidad": El ya aprobado plan de permeabilización entre la marisma de Doñana y el río Guadalquivir permitirá restablecer el funcionamiento estuarino de la desembocadura del Guadalquivir, si bien, se restablecerá el riesgo de entrada de contaminantes a la marisma y especies invasoras. Muy probablemente tenga lugar una diversificación de hábitats de especies reproductoras.

"Política activa de conservación": Deben controlarse los episodios de contaminación en toda la cuenca fluvial y mareal, llevar a cabo una gestión adaptativa que considere la incertidumbre en el proceso de toma de decisiones y convendría que las políticas de conservación y desarrollo económico, tanto locales como europeas, estuvieran basadas en el mantenimiento de los servicios ambientales sin comprometer el funcionamiento de los ecosistemas ni su biodiversidad.

Posteriormente a los análisis individuales por los países, se realizaron talleres de trabajo en los que participaron otros países involucrados en el proyecto, con el fin de producir una síntesis y de identificación de patrones comunes.

\section{CONSIDERACIONES FINALES}

Como se puede apreciar, el proceso de conformación de redes de investigación a escala nacional constituye un verdadero reto ante las limitaciones de recursos materiales y humanos 
de cada país en Iberoamérica. A pesar de las diferencias administrativas y económicas, en ambos casos (y casi de forma generalizada en todas las redes nacionales de ILTER), la iniciativa LTER se enfrenta fundamentalmente a dos retos: la ausencia de estructuras de administración y financiación nacionales para redes conformadas por diferentes instituciones, centros de investigación, universidades, oficinas de gestión, etc. y la dificultad de establecer colaboraciones efectivas entre distintos grupos de investigación a escala nacional. En ambas redes los objetivos son similares, si bien, en la red española se priorizaron como sitios candidatos aquellos bajo una figura de protección dado el interés especial de LTER-España en contribuir con sus actividades a la conservación. Ambas redes representan además las dos estrategias tipo para la implementación de la red:

(1) Aproximación de arriba a abajo, mediante el establecimiento de un comité científico avalado por una institución financiadora de ámbito nacional.

(2) Aproximación de abajo a arriba, mediante la incorporación de miembros autofinanciados con objetivos compartidos promoviendo la sinergia en función de los puntos en común entre los distintos sitios.

Sin embargo, es posible ir avanzando hacia una ciencia más integrada, más útil y sobre todo, más comprometida con la sociedad. Un aspecto clave para la completa implementación de las redes es lograr una verdadera vinculación entre el sector que genera el conocimiento y el que lo pone en práctica. Este es un tema complejo que quisiéramos abordar como última reflexión.

Como plantean Vaughan et al. (2007), las preocupaciones públicas son, cada vez más, asuntos locales y sobre aspectos de sustentabilidad ecológica. Hoy, más que nunca, la sociedad demanda información que le permita enfrentar la severa crisis ambiental. Esta información debe ser aportada de manera más integrada, útil, relevante, comprehensiva y con carácter local. Ya no es posible evaluar el éxito de la ciencia solo en función de cuánta información científica se genera y se hace disponible. Deben establecerse criterios que permitan valorar la calidad de la información generada en función de su utilidad y acierto en la toma de decisiones y de cómo esta información permite mejorar los resultados del manejo y la situación ambiental.

Este planteamiento implica la necesidad de realizar mejoras en la manera como se diseña y se realiza la investigación y el seguimiento ecológicos en nuestros países. Implica también involucrar al investigador en la responsabilidad de informar efectivamente a la sociedad sobre las opciones que tiene para enfrentar la problemática ambiental, así como comunicarle cómo sus políticas y decisiones de manejo repercuten en dicha problemática. El planteamiento también conlleva el incluir "la mejora de resultados" como un criterio adicional del desempeño académico. Estos cambios se presentan difíciles, sobre todo en un sistema científico, de por sí, sujeto a un proceso de evaluación excesiva. Sin embargo, habrá que trabajar en ello si queremos que la ciencia juegue el papel relevante que tiene en la solución de la severa crisis ambiental.

\section{AGRADECIMIENTOS}

Agradecemos la invitación de Christopher Anderson a participar en el encuentro chileno para celebrar el Tercer Aniversario de la Reserva de la Biosfera en Cabo de Hornos y el Lanzamiento de la Red Chilena de Sitios de Estudios Socio-Ecológicos a Largo Plazo. Se agradecen la colaboración de Raúl Ahedo y Salvador Araiza en la preparación del manuscrito. Así también, los autores agradecen los comentarios y sugerencias de Christopher Anderson y de tres revisores anónimos durante el proceso editorial. También se agradece a la red ILTER el marco de trabajo habilitado para permitir los estudios comparados entre redes. La Red Mex-LTER agradece el financiamiento del CONACYT y el INE-SEMARNAT, y el Grupo Chamela del CONACYT y del Programa PAPIIT de la UNAM. La red LTER-España agradece al CSIC, a la Junta de Andalucía, a la Xunta de Galicia, a la Diputación de Barcelona, a la Generalitat de Catalunya y al Organismo Autónomo de Parques Nacionales su apoyo permanente para la implementación de la red española de seguimiento e investigación ecológica a largo plazo. Asimismo agradece al Ministerio de Ciencia e Innovación Español la financiación de las actividades de la red LTEREspaña merced al Proyecto ACI-Comités 
ACI2008-0815 del Programa de Internacionalización de la I+D. La plataforma LTSER-Doñana agradece el financiamiento a través de la red de excelencia ALTER-Net.

\section{LITERATURA CITADA}

BURGOS A, JM MAASS, G CEBALLOS, M EQUIHUA, E JARDEL et al. (2007) La investigación ecológica a largo plazo (LTER) y su proyección en México. Ciencia y Desarrollo (México) 33: 24-31.

BOURGERON P (2008) ILTER ecosystem services assessment project. Interactions among ecosystem services, ecosystem dynamics, and human outcomes and behaviour. URL: http:// www.ilternet.edu/research/ecosystem-servicesassessment (accedido Agosto 30, 2008)

BUSTAMANTE J, PACIOS $\mathrm{F}$, DÍAZ-DELGADO R \& ARAGONÉS D (2009) Predictive models of turbidity and water depth in the Doñana marshes using Landsat TM and ETM+ images. Journal of Environmental Management 90: 2219-2225.

CASADO S \& C MONTES (1991) Estado de conservación de los humedales peninsulares españoles. Quercus 66: 18-26.

CASTILLO JM, L FERNÁNDEZ-BACO, EM CASTELLANOS, CJ LUQUE, ME FIGUEROA \& AJ DAVY (2000) Lower limits of Spartina densiflora and $S$. maritima in a Mediterranean salt marsh determined by different ecophysiological tolerances. Journal of Ecology 88: 801-812.

CHANS JJ \& R DÍAZ-DELGADO (2005) Monitoring and evaluation: The key to the Doñana 2005 restoration project. En: García-Novo F \& C Marín-Cabrera (ed) Doñana, water and biosphere: 319-326. CHG-MMA, Madrid.

COLLINS SL, SM SWINTON, CW ANDERSON, T GRAGSON, NB GRIMM et al. (2007) Integrative science for society and environment: A strategic research initiative. LTER Network Office Publication $\mathrm{N}^{\circ} 23$, National Science Foundation, USA. 35 pp. URL: http://www.lternet.edu/ decadalplan/.

EEA (2005) The European environment. State and outlook 2005. European Environmental Agency, Copenhagen.

EEA (2007) Europe's environment - the fourth assessment. State of the environment report No 1/2007, European Environmental Agency, Copenhagen.

FRANKLIN JF, CS BLEDSOE \& JT CALLAHAN (1990) Contributions of the long-term ecological research program. BioScience 40: 509-523.

GOSZ JR (1996) International long-term ecological research: Priorities and opportunities. Trends in Ecology and Evolution 11: 444.

HABERL H, V WINIWARTER, K ANDERSSON, RU AYRES, C BOONE, A CASTILLO et al. (2006) From LTER to LTSER: Conceptualizing the socioeconomic dimension of long-term socioecological research. Ecology \& Society 11: 13.
HOLLING CS (2002) Adaptive inference for distinguishing credible from incredible patterns in nature. Ecosystems 5: 319-328.

ILTER (2000) The International long-term ecological research network. U.S. LTER Network, University of New Mexico, Albuquerque, New Mexico.

JARDEL EJ, JM MAASS, A CASTILLO, R GARCÍA, L PORTER, J SOSA \& A BURGOS (2008) Manejo de ecosistemas e investigación a largo plazo. Ciencia y Desarrollo (México) 34: 30-37.

LEE KN (1993) Compass \& gyroscope: Integrating science and politics in the environment. Island Press, Washington, DC.

MAASS JM, P BALVANERA, A CASTILLO, GC DAILY, HA MOONEY et al. (2005) Ecosystem services of tropical dry forests: Insights from long-term ecological and social research on the pacific coast of Mexico. Ecology \& Society 10: 17.

MAASS M, M EQUIHUA \& E JARDEL (2008) La Red Mexicana de Investigación Ecológica a Largo Plazo. Ciencia y Desarrollo 34: 215.

MEA (2005) Millennium ecosystem assessment. Ecosystems and human well-being synthesis. Island Press, Washington, DC

METZGER MJ, RGH BUNCE, RHG JONGMAN, CA MÜCHER \& JW WATKINS (2005) A climatic stratification of the environment of Europe. Global Ecology \& Biogeography 14: 549-563.

MIRTL M \& K KRAUZE (2007) Developing a new strategy for environmental research and monitoring: The European Long-term Ecological Research Network's (LTER-Europe) role and perspectives. In: Chmielewski TJ (ed) Nature conservation management: From idea to practical results: 36-52. ALTER-Net. Lublin, Polonia.

NIEVA FJJ, JM CASTILLO, CJ LUQUE \& ME FIGUEROA (2003) Ecophysiology of tidal and non-tidal populations of the invading cordgrass Spartina densiflora: Seasonal and diurnal patterns in a Mediterranean climate. Estuarine, Coastal \& Shelf Science 57: 919-928.

OHL C, K KRAUZE \& C GRUNBUHEL (2007) Towards an understanding of long-term ecosystem dynamics by merging socio-economic and environmental research criteria for long-term socio-ecological research sites selection. Ecological Economics 63: 383-391.

PAIN DJ, A SÁNCHEZ A \& AA MEHARG (1998) The Doñana ecological disaster: Contamination of a world heritage estuarine marsh ecosystem with acidified pyrite mine waste. The Science of the Total Environment 222: 45-54.

RIVERA V, M MAASS, J BENÍTEZ, C CORONADO, J EUÁN et al. (2008) Eco-hidrología y demandas de agua en México. Ciencia y Desarrollo (México) 34: 24-27.

UNEP (2007) Global environmental outlook. Environment for development GEO4. United Nations Environmental Programme, Valetta, Malta.

VAUGHAN H, RB WAIDE, JM MAASS \& E EZCURRA (2007) Developing and delivering scientific information in response to emerging needs. Frontiers in Ecology and the Environment 5: W8-W11. 